\title{
FOLFIRINOX for advanced pancreatic cancer: the Princess Margaret Cancer Centre experience
}

\author{
Muralidharan K Chllamma ${ }^{1}$, Natalie Cook $^{1,2}$, Neesha C Dhani ${ }^{1}$, Kazim Giby ${ }^{1}$, Anna Dodd ${ }^{1}$, Lisa Wang ${ }^{1}$, \\ David W Hedley ${ }^{1}$, Malcolm J Moore ${ }^{1,3}$ and Jennifer J Knox ${ }^{\star, 1}$ \\ ${ }^{1}$ Department of Medical Oncology and Haematology, Princess Margaret Cancer Centre, University Health Network, Toronto \\ M5G 2M9, Canada; ${ }^{2}$ The Christie NHS Foundation Trust/Institute of Cancer Sciences, University of Manchester, Manchester \\ M20 4BX, UK and ${ }^{3}$ President, B.C. Cancer Agency, Vancouver, British Columbia V5Z 4E6, Canada
}

\begin{abstract}
Background: FOLFIRINOX has been shown to significantly increase both overall survival (OS) and progression-free survival (PFS) in metastatic pancreas cancer. There is limited data regarding the treatment of locally advanced pancreatic cancer. We present a retrospective study of patients with both locally advanced and metastatic pancreas cancer using FOLFIRINOX as first-line therapy in our centre.
\end{abstract}

Methods: This is a retrospective review of patients treated with FOLFIRINOX for pancreatic cancer at Princess Margaret Cancer Centre, between December 2011 and July 2014. The primary objective was to evaluate the efficacy and safety of FOLFIRINOX when used with dose modifications.

Results: One hundred two patients were identified; 66 metastatic and 36 locally advanced. Sixty-eight per cent of patients initiated treatment with a dose reduction. The median (95\% Cl) OS in the metastatic group was 13.1 (6.3-16.1) months with full dose and 12.9 (10.3-30.1) months with modified dose. The median (95\% Cl) OS in the locally advanced group was 11.1 (6.1-not reached) months with full dose and 23 (not reached-not reached) months with modified dose. The median (95\% Cl) PFS in the metastatic group was 6.2 (4.9-15.2) months with full dose and 8.7 (5.7-12.9) months with modified dose. The median (95\% Cl) PFS in the locally advanced group was 11.1 (3.1-not reached) months with full dose and 10.4 (6.8-not reached) months with modified dose. Grade 3/4 haematologic adverse events were observed in 43\% of patients. Grade 3/4 nonhaematologic adverse events were observed in $28 \%$ of patients. Patient well-being significantly improved from baseline to cycle $4(P=0.002)$.

Conclusions: Efficacy was achievable with dose-modified FOLFIRINOX in daily setting. The safety of FOLFIRINOX remains a concern with a high rate of grades 3 and 4 neutropaenia despite dose reduction.

Pancreatic ductal adenocarcinoma (PDA) is one of the most lethal human cancers because of late presentation, early metastases and resistance of tumour cells to conventional treatments such as radiation and chemotherapy (Haeno et al, 2012; Tuveson and Neoptolemos, 2012; Sohal et al, 2014). It is the tenth most common cancer in both men and women in Canada; however it is the fourth leading cause of cancer-related death in both sexes. Approximately
$10 \%$ of patients are diagnosed with operable disease with a 5 -year survival rate of around 20\% (Mayo et al, 2012). Unfortunately more than half of patients present with metastases, for which the 5 -year survival is only 2\% (Malvezzi et al, 2013). Until recently, standard chemotherapy used in both adjuvant and palliative settings has been gemcitabine; however treatment with gemcitabine alone has low response rates and minimal effect on survival

*Correspondence: Dr JJ Knox; E-mail: jennifer.knox@uhn.ca

Received 23 January 2016; revised 30 June 2016; accepted 4 July 2016; published online 28 July 2016

(c) 2016 Cancer Research UK. All rights reserved 0007 - 0920/16 
(Burris et al, 1997; di Marco et al, 2010; Renouf and Moore, 2010). Therefore improved chemotherapeutic or targeted therapy regimens are desperately needed.

Recently, two trials investigating combined chemotherapy use in metastatic PDA have reported encouraging results for improving overall survival (OS; Conroy et al, 2011; von Hoff et al, 2013). One of these trials used combination chemotherapy with FOLFIRINOX (oxaliplatin, irinotecan, fluorouracil and leucovorin) (Conroy et al, 2011). This was the first chemotherapy regimen tested against standard gemcitabine to significantly improve survival in metastatic PDA. This trial (ACCORD 11) revealed a median overall survival on FOLFIRINOX of 11.1 months compared with 6.8 months in the gemcitabine arm, producing the longest seen survival advantage observed in a phase III clinical trial for metastatic pancreatic carcinoma. Although there were higher rates of adverse events (AEs) on FOLFIRINOX, patients in this group also reported a better quality of life (QoL; Gourgou-Bourgade et al, 2013). FOLFIRINOX has also been shown to be a more cost effective first-line treatment compared with first-line gemcitabine (Attard et al, 2014).

Since the publication of ACCORD trial, the FOLFIRINOX regimen is increasingly used at institutions worldwide to treat metastatic PDA. Due to the higher rate of AEs, and the highly selected patient population in the original trial, it is difficult to say whether this chemotherapy regimen should be used in all patients with advanced pancreatic cancer. In a retrospective study reviewing 100 consecutive cases of metastatic PDA it was found that only 26 patients fulfilled the ACCORD study eligibility criteria (Ho et al, 2015). It is also not known whether dose reductions compromise the efficacy of FOLFIRINOX, moreover there is limited data regarding the efficacy of FOLFIRINOX in locally advanced pancreatic cancer (LAPC) (Hosein et al, 2012; Faris et al, 2013; Gunturu et al, 2013; Blazer et al, 2015). Recent retrospective reviews and publication have revealed varying efficacy of modified FOLFIRINOX regimens in small numbers of patients (Peddi et al, 2012; Faris et al, 2013; Gunturu et al, 2013; Mahaseth et al, 2013). Therefore we conducted a retrospective review of patients with metastatic or unresectable pancreatic cancer treated with FOLFIRINOX at the Princess Margaret Cancer Centre (PM).

\section{MATERIALS AND METHODS}

Design. All patients with a diagnosis of locally advanced or metastatic pancreatic cancer who began treatment with FOLFIRINOX at PM were identified by searching the cancer centre pharmacy database under a study protocol approved by the Research and Ethics Committee. This includes a list of patients that have received the FOLFIRINOX regimen since it was approved by Cancer Care Ontario. The primary objective of this study was to evaluate the efficacy and safety of the FOLFIRINOX regimen. A secondary objective was to document preliminary evidence of the efficacy of FOLFIRINOX in patients with LAPC. Locally advanced pancreatic cancer included patients who were unresectable at presentation, defined as patients with metastases to lymph nodes beyond the resection field, $>180^{\circ}$ encasement of the superior mesenteric artery, unreconstructable superior mesenteric vein or portal vein occlusion, aortic invasion or coeliac encasement (Callery et al, 2009). PM is a University Teaching Hospital and the regional referral centre for patients with operable and inoperable pancreatic adenocarcinoma. Patients were supervised by four staff medical oncologists.

The list of patients who received FOLFIRINOX was transferred to a secure database, and patient's electronic records and chemotherapy records were examined to record the following information: date of diagnosis, stage and clinico-pathological features of cancer, ECOG performance status, treatment intent, number of cycles received including percentage of starting doses of each drug and subsequent dose reductions, main AEs in the first two cycles of treatment, any grade $3 / 4$ AEs, hospitalisations during treatment, objective radiological responses, progression-free survival (PFS) and OS, Distress Assessment and Response Tool (DART) assessments (using the scores for tiredness, pain and well-being) and subsequent treatment regimens. DART is a self-administered computerised survey including screens for physical symptoms (Edmonton Symptom Assessment System or ESAS), anxiety and depression (Bruera et al, 1991; Watanabe et al, 2012). Patients complete DART as part of routine clinical care throughout their cancer trajectory. Only patients who completed one full cycle of FOLFIRINOX were included for analysis. Adverse events were graded using National Cancer Institute Common Toxicity Criteria (CTCAE version 4.0).

Chemotherapy regimen. Full-dose FOLFIRINOX was administered as per the phase III trial of FOLFIRINOX (Conroy et al, 2011), with 5-FU administered as a bolus of $400 \mathrm{mg} \mathrm{m}^{-2}$, bolus leucovorin $400 \mathrm{mg} \mathrm{m}^{-2}$, followed by continuous infusion at $1200 \mathrm{mg} \mathrm{m}^{-2}$ per day for $46 \mathrm{~h}$, oxaliplatin $85 \mathrm{mg} \mathrm{m}^{-2}$ and irinotecan $180 \mathrm{mg} \mathrm{m}^{-2}$. Patients routinely received ondansetron and dexamethasone for emesis prophylaxis pre-chemo and for 2 days post chemotherapy. Atropine was given to patients who had cholinergic reactions from irinotecan. Because of funding issues with the use of G-CSF in a non-curative setting, and documented toxicity issues with this regimen, many of our patients were treated with attenuated doses. Therefore prophylactic G-CSF was not administered, however when patients did subsequently receive this it was recorded. Choices regarding dosing modifications were determined by the individual treating oncologist based on age, general condition and co-morbid medical illnesses and recorded in our database.

Assessments. Patients were initially evaluated for toxicities at the start of each cycle with history, performance status, complete blood counts, liver and electrolyte blood tests. If stable after two cycles, they would then be assessed on alternate cycles. Response assessment with imaging was obtained approximately every four cycles of treatment. For all patients, treatment was continued until disease progression, patient preference or limiting toxicities. Patients were assessed for tumour response by the treating oncologist according to the Response Evaluation Criteria in Solid Tumors guidelines (RECIST, version 1.1).

Statistics. Progression-free survival was calculated from the date of the first dose of FOLFIRINOX to the earliest of the following: date of radiographic progression (local or metastatic), detection of metastatic disease at surgical exploration or death. PFS and OS estimates were obtained using the Kaplan-Meier method. The association between dose and stage with PFS and OS was carried out using log-rank test and Cox proportional hazard model. Quality of life scores were compared with baseline using Wilcoxon signed rank test. Summary statistics were used to describe the results. SAS version 9.2 was used for all the analysis.

\section{RESULTS}

Patient characteristics. Between December 2011 and April 2014, a total of 102 patients began treatment with FOLFIRINOX at Princess Margaret Cancer Centre, including 66 with metastatic disease and 36 with LAPC. Patients receiving at least one dose of FOLFIRINOX were included and the database was closed for analysis on 8 July 2014. Patient demographics and baseline characteristics are shown in Table 1 . Median age of patients initiating treatment was 64 years (range 28-76), 3\% of patients 
Table 1. Demographics and baseline characteristics of patients

\begin{tabular}{|l|c|}
\hline Characteristic & $\mathbf{n}(\%)$ \\
\hline Median age at diagnosis in years (range) & $64(28-76)$ \\
\hline Sex & \\
$\quad$ Male & $57(55.9)$ \\
$\quad$ Female & $45(44.1)$ \\
\hline ECOG Performance Status score & \\
0 & $48(47.1))$ \\
1 & $49(48)$ \\
2 & $5(4.9)$ \\
\hline Pancreatic tumour location & $73(71.6)$ \\
Proximal—Head, neck or uncinated process & $29(28.4)$ \\
Distal—body or tail & \\
\hline Biliary stent placed before start of treatment & $46(45.1)$ \\
Yes & $56(54.9)$ \\
No & \\
\hline Bilirubin at start of treatment & $97(95.1)$ \\
$<1.5$ UNL & $5(4.9)$ \\
\hline 1.5 UNL & \\
\hline Abbreviations: ECOG = Eastern Cooperative Oncology Group; UNL =upper limit of the \\
normal range.
\end{tabular}

Table 2. Treatment duration and dose modifications

\begin{tabular}{|l|c|}
\hline Cycles & Number \\
\hline Median & 6 \\
\hline Range & $1-31$ \\
\hline$>3$ & 77 \\
\hline 3 & 4 \\
\hline 2 & 9 \\
\hline 1 & 12 \\
\hline Dose modifications & $n(\%)$ \\
\hline Started with full dose of all drugs & $33(32.4)$ \\
\hline Started with reduced dose of 1 or more drugs & $69(67.6)$ \\
\hline Reduction or omission of 5-FU bolus & $93 \%$ \\
\hline Reduction in irinotecan & $88 \%$ \\
\hline Reduction in oxaliplatin & $68 \%$ \\
\hline Reduction infusional 5-FU & $66 \%$ \\
\hline
\end{tabular}

were over 75 years. Five per cent of our patients had performance status 2 ECOG and a further $5 \%$ had bilirubin $>1.5$ ULN at the start of treatment.

Dose modifications. Chemotherapy dose modifications were left to the discretion of the treating physician. Dose modifications and chemotherapy cycle details are shown in Table 2 and Figure 1. Only 33 (32.4\%) of 102 patients received full doses of all drugs in the regimen with their first cycle. In the remaining $69(67.6 \%)$ of patients, dose reductions with first cycle were as follows: Bolus FU was reduced or omitted in $93 \%$, irinotecan was reduced in $88 \%$, oxaliplatin was dose reduced in $68 \%$ and infusional $\mathrm{FU}$ was reduced in $66 \%$. The median number of cycles administered was 6 (range 1-31). Twenty-five (24.5\%) patients received three or less chemotherapy cycles either due to disease progression or poor tolerance. Twelve patients received only one cycle; six stopped due to progression and six due to poor tolerance. Nine patients received two cycles; three stopped due to progression and six due to poor tolerance. Three patients received three cycles; two stopped due to progression and one due to death.

Adverse events and hospitalisations. Treatment-related grade 3 or 4 adverse events are summarised in Table 3 . Grade 3 or 4 haematologic adverse events were observed in $43 \%$ of patients, with

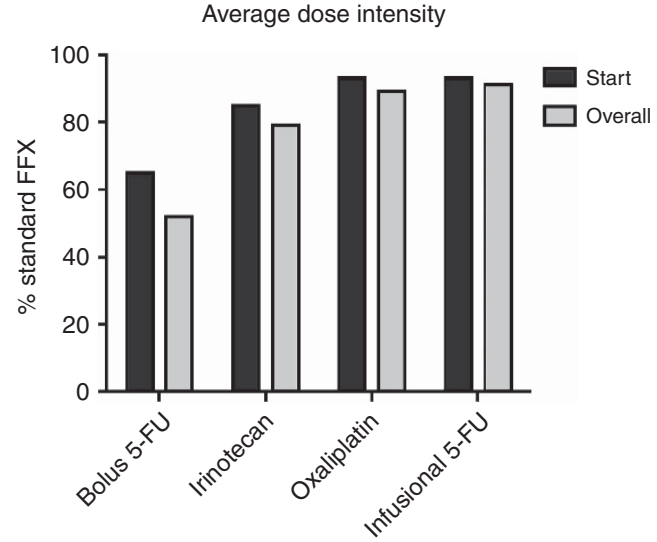

Figure 1. Bar chart showing starting and overall average dose intensity for bolus 5-Fluorouracil, oaxaliplatin, irinotecan and infusional 5-fluorouracil.

Table 3. Common grade 3 or 4 adverse events

\begin{tabular}{|l|c|}
\hline Event & $\mathbf{n}(\%)$ \\
\hline Haematologic & $44(43.1)$ \\
\hline Neutropenia & $38(37.3)$ \\
\hline Febrile neutropenia & $6(5.9)$ \\
\hline Thrombocytopenia & $2(2)$ \\
\hline Anaemia & $3(2.9)$ \\
\hline Non-haematologic & $29(28.4)$ \\
\hline Vomiting & $19(18.6)$ \\
\hline Nausea & $16(15.7)$ \\
\hline Diarrhoea & $16(15.7)$ \\
\hline Fatigue & $1(1)$ \\
\hline Loss of appetite & $1(1)$ \\
\hline
\end{tabular}

febrile neutropenia in $6 \%$. Only $13(12.7 \%)$ of the patients received G-CSF support, mostly as secondary prophylaxis. Grade 3 or 4 non-haematologic toxicities were observed in $28 \%$ of patients with vomiting (19\%), nausea (16\%) and diarrhoea (16\%) being the most common. Eighteen per cent of patients had treatment related hospitalisation. Five per cent were hospitalised due to neutropenic sepsis and $13 \%$ due to GI toxicity or dehydration. There was one toxic death attributable to FOLFIRINOX. The patient whose death was attributed to FOLFRINOX tolerated the first two cycles well but died as a result of toxicities following the third cycle. This patient was hospitalised with severe diarrhoea and hyponatremia 2 days following the third cycle. His condition improved initially but then, he developed respiratory distress on the fourth day after admission and died the following day. The most likely cause of death is thought to be pulmonary embolism.

Response by RECIST and CA 19.9. Tumour response was assessed by RECIST criteria in all patients who had completed four cycles of FOLFIRINOX. Disease control was achieved in 58 (56.8\%) patients of the entire group, with partial response (PR) in $14(13.7 \%)$ patients and stable disease (SD) in $44(43.1 \%)$ patients. Eleven (16.6\%) of metastatic pancreatic patients had a PR and 35 (53\%) had SD.

Two patients with LAPC were considered to be borderline resectable and treated with FOLFIRNOX as neoadjuvant chemotherapy. One had a successful surgery with Whipple resection and wedge resection of a solitary liver metastasis detected at the time of surgery. The other had progression of disease locally and 
was treated with palliative radiotherapy. Ten patients with LAPC, who were considered unresectable because of arterial involvement, were treated with FOLFIRINOX as sort of 'primary chemotherapy'. Of these, three patients underwent a successful surgery with arterial resection and reconstruction. One patient with locally advanced disease was treated initially with palliative intent, but had a very good response leading to surgery. So, out of the six patients with LAPC who underwent surgery after primary chemotherapy, five had a successful R0 surgical resection. One was unresectable, due to pre-operatively undetected peritoneal disease.

Patients CA19-9 results were assessed at baseline, and after two and four cycles of FOLFIRINOX. Eighty two per cent of patients had tumours that secreted CA19.9. In 14 patients there was insufficient data to assess any correlation between CA19-9 and response rate. However in those where data were available at each time-point there was a clear correlation between responding CA199 values and radiological response rate after four cycles of treatment. Patients with partial responses always had significant responses in their CA19-9 results.

Progression-free and overall survival. The median OS was 12.9 months in metastatic patients and 23 months in LAPC. The median PFS was 8.7 months in the metastatic and 11.1 months in the LAPC. Starting dose and dose intensity were not associated with OS or PFS in the univariate analysis. There was no significant difference in PFS (10.9 months $v s 10.3$ months; $P$-value $=0.60)$ or OS (11.1 months vs 14.0 months; $P$-value $=0.19)$ between the full starting dose and reduced starting dose groups, respectively. The median (95\% CI) OS in the metastatic group was 13.1 (6.3-16.1) months with full dose and $12.9(10.3-30.1)$ months with modified dose (Figure $2 \mathrm{~A})$. The median $(95 \% \mathrm{CI})$ OS in the locally advance group was 11.1 (6.1-not reached) months with full dose and 23 (not reached-not reached) months with modified dose (Figure 2B). The median (95\% CI) PFS in the metastatic group was 6.2 (4.915.2) months with full dose and 8.7 (5.7-12.9) months with modified dose (Figure 3A). The median (95\% CI) PFS in the locally advanced group was 11.1 (3.1-not reached) months with full dose and 10.4 (6.8-not reached) months with modified dose (Figure 3B).

Second-line therapy. At the time of closure of database for analysis, forty-six patients had received second-line therapy after FOLFIRINOX. The most common regimen to be used as second line was single agent Gemcitabine in 32 patients (69.6\%). Gemcitabine and nab-paclitaxel was used in 7 (15.2\%). Two patients who were BRCA positive were treated with Cisplatin and Gemcitabine combination. Three patients were treated on phase 1 trials after FOLFIRINOX.

Quality of life. Patient's well-being was assessed using the DART questionnaire on every visit. Three factors; well-being, pain and tiredness were chosen for analysis from the 10-point score. Patients' well-being scores were significantly improved from baseline to cycle 4 (after 2 months of treatment) $(P=0.002)$. However, pain and tiredness at cycle 4 were not significantly different from baseline. None of the changes in these scores were significantly associated with OS or PFS.

\section{DISCUSSION}

In this study, the median OS in metastatic patients treated with FOLFIRINOX was an encouraging 12.9 months and the median PFS was 8.7 months. This was in spite of our study having a significant proportion (67.6\%) of patients commencing treatment with a dose modification. Few other studies have investigated the impact of dose modifications on response to the FOLFIRINOX regimen. A study of 36 patients with metastatic
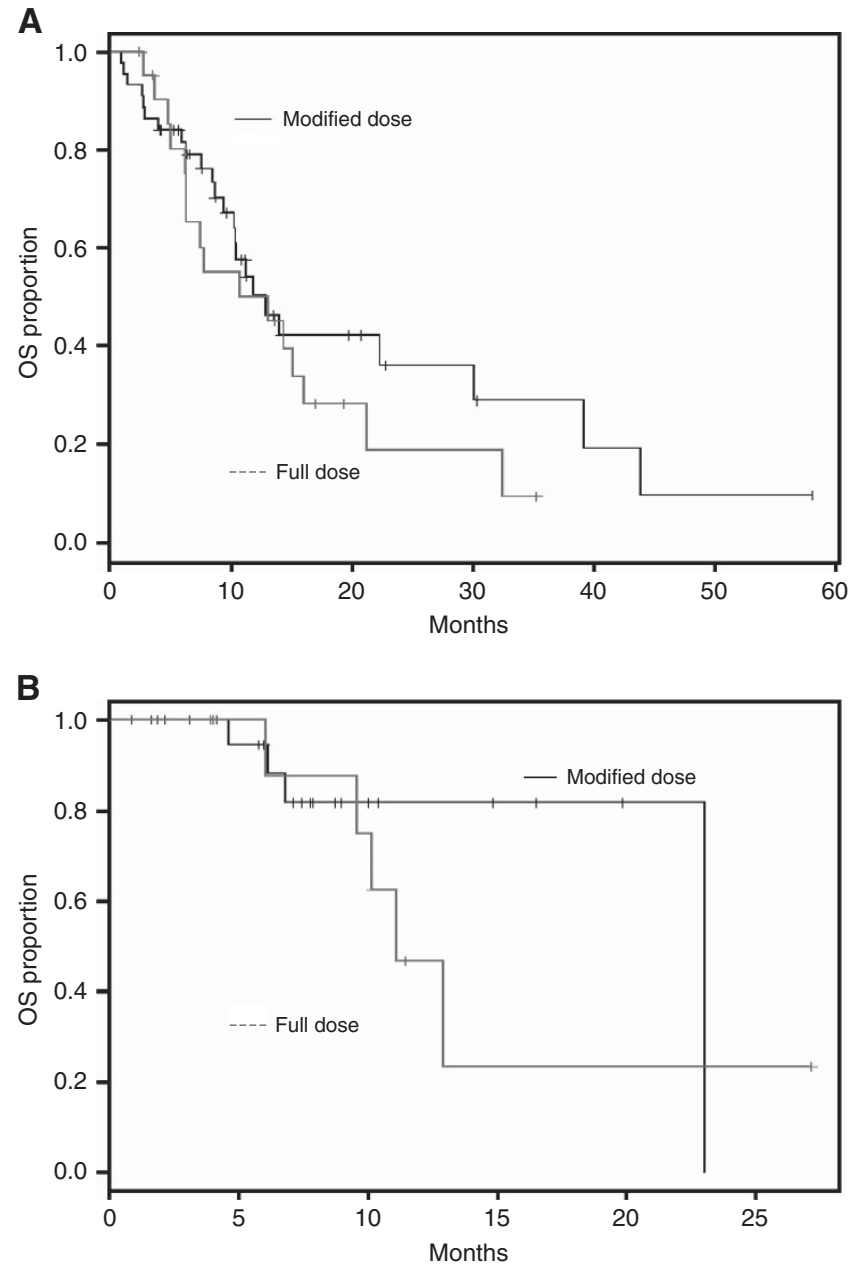

Figure 2. Kaplan-Meier plots of overall survival. (A) Median OS with full dose and modified dose in metastatic patients. Median (95\% Cl) OS with full dose was 13.1 (6.3-16.1) months and with modified dose was 12.9 (10.3-30.1). (B) Median OS with full dose and modified dose in non-metastatic patients. Median $(95 \% \mathrm{Cl})$ OS with full dose was 11.1 (6.1-not reached) months and with modified dose 23(not reached-not reached) months.

pancreatic adenocarcinoma (Mahaseth et al, 2013) examined modified FOLFIRINOX, and reported a median OS of 9 months and PFS of 8.5 months. Another small study of 19 patients (Gunturu et al, 2013) in a similar patient population reported median PFS of 9.9 months and median OS was not reached at the time of reporting.

In our study the median OS in LAPC was 23 months and the PFS was 11.1 months. In a study performed at Massachusetts General Hospital (Faris et al, 2013), which included 22 patients with LAPC, the median PFS was 11.7 months and 5 out of 22 (23\%) patients were able to undergo R0 resections following neoadjuvant FOLFIRINOX and chemoradiation. In this study prophylactic G-CSF was used in all patients. Mahaseth et al (2013) reported a median OS of 17.8 months and PFS of 13.7 months in their series of 24 patients with LAPC. Gunturu reported PFS of 11.2 months in his study of 16 patients of LAPC (Gunturu et al, 2013). Primary prophylaxis with G-CSF was used in both studies. G-CSF was used only as secondary prophylaxis and infrequently in our group. In most patients who developed grade $3 / 4$ neutropenia further chemotherapy was administered with dose reductions, rather than using prophylactic G-CSF. The results of our study are concurrent with several other studies performed in LAPC (Gunturu et al, 2013; Mahaseth et al, 2013). 

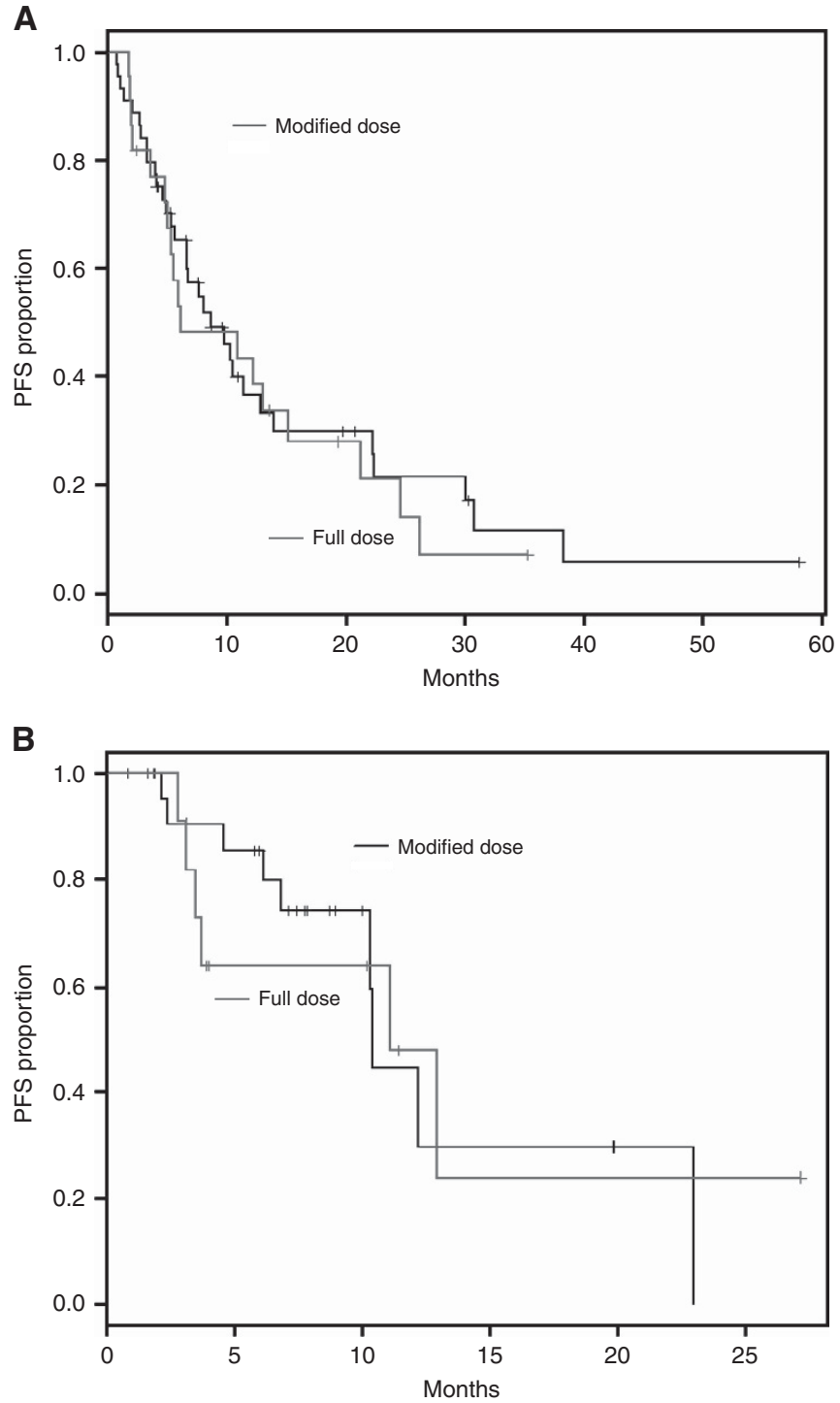

Figure 3. Kaplan-Meier plots of progression-free survival. (A) Median PFS with full dose and modified dose in metastatic patients. Median $(95 \% \mathrm{Cl})$ OS with full dose was 6.2 (4.9-15.2) months and with modified dose was 8.7 (5.7-12.9) months. (B) Median PFS with full dose and modified dose in non-metastatic patients. Median (95\% Cl) OS with full dose was 11.1 (3.1-not reached) months and with modified dose 10.4 (6.8-not reached) months.

A multicentre experience of six Canadian institutions, noted a median OS of 14 months in metastatic patients and 21.5 months in locally advanced pancreatic patients (Amirealut et al, 2014). Fifty-five patients ( 30 metastatic and 25 locally advanced) were included in this study. Similarly the Royal Marsden group reported a median OS of 18.4 months in LAPC (27 patients) and 10.4 months in MPC (22 patients). Dose reduction of one or more drugs was made in $74 \%$ of patients in this study (Moorcraft et al, 2014). A study from South Asia with 23 MPC patients observed a median OS of 10.4 months and median PFS of 7.3 months (Zahir and Jabbar, 2015). However, an Irish study with 25 patients, noted a lower PFS and OS of 3.9 months and 7 months, respectively (Walsh, 2014). Over 50\% in this cohort required a dose reduction and they concluded that FOLFIRINOX while promising in the clinical trial setting may not translate to routine clinical practice. A recent meta-analysis of 13 studies, with a total of 253 patients reported a R0 resection of $40 \%$ after neo-adjuvant
FOLFIRINOX-based therapy in patients with borderline resectable/unresectable pancreatic adenocarcinoma (Petrelli et al, 2015).

Evaluating the response by following the changes in tumour dimensions, as assessed by CT scan/MRI is often challenging (Donahue et al, 2011). Changes in tumour marker CA 19.9 is often helpful in such situations (Pelzer et al, 2013). In a study of 129 patients with borderline resectable tumours, imaging suggested that only $1 \%$ had been down staged, $78 \%$ had no change and $21 \%$ had progressive disease (Katz et al, 2012). However, $66 \%$ were able to undergo resection with 95\% R0 resections. In our study there was a clear correlation between responding CA19-9 values and radiological response rate after four cycles of treatment.

Our study is the largest single institute retrospective study to examine the efficacy of reduced dose FOLFIRINOX in metastatic and locally advanced pancreatic cancer. About two-thirds of our patients received their first and subsequent cycles of chemotherapy with reduced doses. Nonetheless, the efficacy did not seem to be compromised by these dose reductions, given there were no significant differences in PFS or OS between the full starting dose and reduced starting dose groups. Although response rate was lower than that of other reports likely due to off-study ascertainment issue, the more clinically relevant outcomes of OS and PFS were comparable to other studies utilising dose modifications (Peddi et al, 2012; Gunturu et al, 2013; Faris et al, 2013; Mahaseth et al, 2013; Blazer et al, 2015). The toxicities associated with FOLFIRINOX have tempered the enthusiasm for its use in full dose in community as well as in academic centres (Amirealut et al, 2014). Our study has shown that modest dose reductions do not appear to compromise the efficacy while reducing the toxicities associated with the regimen. We observed a significantly lower incidence of grade $3 / 4$ fatigue (1\%) and no incidence of grade $3 / 4$ peripheral neuropathy. Safety has to be taken into consideration and we did not use prophylactic G-CSF routinely in our patients, although the incidence of grade $3 / 4$ neutropenia and febrile neutropenia were relatively low. The treatment adverse event-related hospitalisation rate in our study was $18 \%$. Much higher rates have been reported by others (Peddi et al, 2012; Amirealut et al, 2014) in spite of many patients receiving colony-stimulating factors as primary prophylaxis. ACCORD III was dominated by tumours in the body and tail of the pancreas. Our rates for grade $3 / 4$ toxicity remained low even for patients with a high rate of biliary stenting. In our study there was one treatment related death, which was attributable to FOLFIRINOX.

Health-related QoL was maintained on FOLFIRINOX, highlighting the fact that in advanced pancreatic adenocarcinoma, QoL is influenced more by disease-related symptoms than treatment-related toxicity. It is encouraging to note results from our single-centre experience are consistent with results from other smaller studies utilising dose modifications. However, there are clearly limitations with a retrospective single-centre review. In our study there was no standardised dose modifications made by the physicians, there was no independent radiological reviews and no standardised method to collect toxicity information. There was also heterogeneity of the cohort as we assessed locally advanced and metastatic pancreatic cancers together and one-third of patients did commence treatment at full dose. We also cannot discount the impact second-line therapy may have had on PFS and OS.

In summary, this single-centre experience suggests that FOLFIRINOX with dose modifications, in the absence of G-CSF can be administered safely in a real-world setting. However, to directly compare these results to full dose FOLFIRINOX treatment a randomised trial would be recommended, incorporating QoL assessments. 


\section{CONFLICT OF INTEREST}

The authors declare no conflict of interest.

\section{REFERENCES}

Amirealut C, Beaudet J, Gaudet G, Raymond N, Ayoub J-PM, Letourneau R, Loungnarath R, Tehfe MA, Aubin F (2014) FOLFIRINOX in the real world setting: the multicentric experience of six Canadian Institutions. J Clin Oncol 32(suppl 3): abstract 367.

Attard CL, Brown S, Alloul K, Moore MJ (2014) Cost-effectiveness of folfirinox for first-line treatment of metastatic pancreatic cancer. Curr Oncol 21: e41-e51.

Blazer M, Wu C, Goldberg RM, Phillips G, Schmidt C, Muscarella P, Wuthrick E, Williams TM, Reardon J, Ellison EC, Bloomston M, Bekaii-Saab T (2015) Neoadjuvant modified (m) FOLFIRINOX for locally advanced unresectable (LAPC) and borderline resectable (BRPC) adenocarcinoma of the pancreas. Ann Surg Oncol 22: 1153-1159.

Bruera E, Kuehn N, Miller MJ, Selmser P, Macmillan K (1991) The Edmonton Symptom Assessment System (ESAS): a simple method for the assessment of palliative care patients. J Palliat Care 7: 6-9.

Burris HA 3rd, Moore MJ, Andersen J, Green MR, Rothenberg ML, Modiano MR, Cripps MC, Portenoy RK, Storniolo AM, Tarassoff P, Nelson R, Dorr FA, Stephens CD, von Hoff DD (1997) Improvements in survival and clinical benefit with gemcitabine as first-line therapy for patients with advanced pancreas cancer: a randomized trial. J Clin Oncol 15: 2403-2413.

Callery MP, Chang KJ, Fishman EK, Talamonti MS, William Traverso L, Linehan DC (2009) Pretreatment assessment of resectable and borderline resectable pancreatic cancer: expert consensus statement. Ann Surg Oncol 16: $1727-1733$

Conroy T, Desseigne F, Ychou M, Bouche O, Guimbaud R, Becouarn Y, Adenis A, Raoul JL, Gourgou-Bourgade S, de la Fouchardiere C, Bennouna J, Bachet JB, Khemissa-Akouz F, Pere-Verge D, Delbaldo C, Assenat E, Chauffert B, Michel P, Montoto-Grillot C, Ducreux M, Groupe Tumeurs Digestives of Unicancer, PRODIGE Intergroup. (2011) FOLFIRINOX versus gemcitabine for metastatic pancreatic cancer. $N$ Engl J Med 364 $1817-1825$.

di Marco M, di Cicilia R, Macchini M, Nobili E, Vecchiarelli S, Brandi G, Biasco G (2010) Metastatic pancreatic cancer: is gemcitabine still the best standard treatment? (Review). Oncol Rep 23: 1183-1192.

Donahue TR, Isacoff WH, Hines OJ, Tomlinson JS, Farrell JJ, Bhat YM, Garon E, Clerkin B, Reber HA (2011) Downstaging chemotherapy and alteration in the classic computed tomography/magnetic resonance imaging signs of vascular involvement in patients with pancreaticobiliary malignant tumors: influence on patient selection for surgery. Arch Surg 146: 836-843.

Faris JE, Blaszkowsky LS, Mcdermott S, Guimaraes AR, Szymonifka J, Huynh MA, Ferrone CR, Wargo JA, Allen JN, Dias LE, Kwak EL, Lillemoe KD, Thayer SP, Murphy JE, Zhu AX, Sahani DV, Wo JY, Clark JW, Fernandez-Del Castillo C, Ryan DP, Hong TS (2013) FOLFIRINOX in locally advanced pancreatic cancer: the Massachusetts General Hospital Cancer Center experience. Oncologist 18: 543-548.

Gourgou-Bourgade S, Bascoul-Mollevi C, Desseigne F, Ychou M, Bouche O, Guimbaud R, Becouarn Y, Adenis A, Raoul JL, Boige V, Berille J, Conroy T (2013) Impact of FOLFIRINOX compared with gemcitabine on quality of life in patients with metastatic pancreatic cancer: results from the PRODIGE 4/ACCORD 11 randomized trial. J Clin Oncol 31: 23-29.

Gunturu KS, Yao X, Cong X, Thumar JR, Hochster HS, Stein SM, Lacy J (2013) FOLFIRINOX for locally advanced and metastatic pancreatic cancer: single institution retrospective review of efficacy and toxicity. Med Oncol 30: 361.

Haeno H, Gonen M, Davis MB, Herman JM, Iacobuzio-Donahue CA, Michor F (2012) Computational modeling of pancreatic cancer reveals kinetics of metastasis suggesting optimum treatment strategies. Cell 148: 362-375.

Ho MY, Kennecke HF, Renouf DJ, Cheung WY, Lim HJ, Gill S (2015) Defining eligibility of FOLFIRINOX for first-line metastatic pancreatic adenocarcinoma (MPC) in the Province of British Columbia: a population-based retrospective study. Am J Clin Oncol; e-pub ahead of print 9 July 2015; doi:10.1097/COC.0000000000000205.

Hosein PJ, Macintyre J, Kawamura C, Maldonado JC, Ernani V, Loaiza-Bonilla A, Narayanan G, Ribeiro A, Portelance L, Merchan JR, Levi JU, Rocha-Lima CM (2012) A retrospective study of neoadjuvant FOLFIRINOX in unresectable or borderline-resectable locally advanced pancreatic adenocarcinoma. BMC Cancer 12: 199.

Katz MH, Fleming JB, Bhosale P, Varadhachary G, Lee JE, Wolff R, Wang H, Abbruzzese J, Pisters PW, Vauthey JN, Charnsangavej C, Tamm E, Crane CH, Balachandran A (2012) Response of borderline resectable pancreatic cancer to neoadjuvant therapy is not reflected by radiographic indicators. Cancer 118: 5749-5756.

Mahaseth H, Brutcher E, Kauh J, Hawk N, Kim S, Chen Z, Kooby DA, Maithel SK, Landry J, El-Rayes BF (2013) Modified FOLFIRINOX regimen with improved safety and maintained efficacy in pancreatic adenocarcinoma. Pancreas 42: 1311-1315.

Malvezzi M, Bertuccio P, Levi F, La Vecchia C, Negri E (2013) European cancer mortality predictions for the year 2013. Ann Oncol 24: 792-800.

Mayo SC, Nathan H, Cameron JL, Olino K, Edil BH, Herman JM, Hirose K, Schulick RD, Choti MA, Wolfgang CL, Pawlik TM (2012) Conditional survival in patients with pancreatic ductal adenocarcinoma resected with curative intent. Cancer 118: 2674-2681.

Moorcraft SY, Khan K, Peckitt C, Watkins D, Rao S, Cunningham D, Chau I (2014) FOLFIRINOX for locally advanced or metastatic pancreatic ductal adenocarcinoma: the Royal Marsden experience. Clin Colorectal Cancer 13: 232-238.

Peddi PF, Lubner S, McWilliams R, Tan BR, Picus J, Sorscher SM, Suresh R, Lockhart AC, Wang J, Menias C, Gao F, Linehan D, Wang-Gillam A (2012) Multi-institutional experience with FOLFIRINOX in pancreatic adenocarcinoma. JOP 13: 497-501.

Pelzer U, Hilbig A, Sinn M, Stieler J, Bahra M, Dorken B, Riess H (2013) Value of carbohydrate antigen 19-9 in predicting response and therapy control in patients with metastatic pancreatic cancer undergoing first-line therapy. Front Oncol 3: 155.

Petrelli F, Coinu A, Borgonovo K, Cabiddu M, Ghilardi M, Lonati V, Aitini E, Barni S. Gruppo Italiano per lo Studio dei Carcinomi Dell'apparato, D. (2015) FOLFIRINOX-based neoadjuvant therapy in borderline resectable or unresectable pancreatic cancer: a meta-analytical review of published studies. Pancreas 44: 515-521.

Renouf D, Moore M (2010) Evolution of systemic therapy for advanced pancreatic cancer. Expert Rev Anticancer Ther 10: 529-540.

Sohal DP, Walsh RM, Ramanathan RK, Khorana AA (2014) Pancreatic adenocarcinoma: treating a systemic disease with systemic therapy. $J$ Natl Cancer Inst 106: dju011.

Tuveson DA, Neoptolemos JP (2012) Understanding metastasis in pancreatic cancer: a call for new clinical approaches. Cell 148: 21-23.

von Hoff DD, Ervin T, Arena FP, Chiorean EG, Infante J, Moore M, Seay T, Tjulandin SA, Ma WW, Saleh MN, Harris M, Reni M, Dowden S, Laheru D, Bahary N, Ramanathan RK, Tabernero J, Hidalgo M, Goldstein D, van Cutsem E, Wei X, Iglesias J, Renschler MF (2013) Increased survival in pancreatic cancer with nab-paclitaxel plus gemcitabine. N Engl J Med 369: 1691-1703.

Walsh EMA, Teo MY, Blaskova S, Donnellan PP, Keane MM, Leonard GD (2014) FOLFORINOX in pancreatic cancer : Can results be reproduced outside the clinic trial setting? J Clinical Oncol 32(suppl 15): e15236.

Watanabe SM, Nekolaichuk CL, Beaumont C (2012) The Edmonton Symptom Assessment System, a proposed tool for distress screening in cancer patients: development and refinement. Psychooncology 21: 977-985.

Zahir MN, Jabbar AA (2015) Metastatic pancreatic carcinoma and experience with FOLFIRINOX - a cross sectional analysis from a developing country. Asian Pac J Cancer Prev 16: 6001-6006.

This work is published under the standard license to publish agreement. After 12 months the work will become freely available and the license terms will switch to a Creative Commons AttributionNonCommercial-Share Alike 4.0 Unported License. 